\title{
Treatment of traumatic facial paralysis with mechanical stimulus - hyperboloid: case report
}

\author{
Fabiana Augusto Novo Borghi ${ }^{1}$, Rosana Queiroz ${ }^{2}$, José Ricardo Gurgel Testa ${ }^{3}$ \\ ${ }^{1}$ Functional Jaw Orthopedics, Santa Bárbara d' Oeste, SP, Brazil \\ ${ }^{2,3}$ Unifesp - EPM, São Paulo, SP, Brazil \\ ${ }^{1}$ Corresponding author \\ E-mail: ${ }^{1}$ fabiananovoborghi@gmail.com, ${ }^{2}$ rosanaqueiroz@msn.com, ${ }^{3}$ drtesta13@terra.com.br
}

Received 2 May 2021; received in revised form 23 August 2021; accepted 7 September 2021 DOI https://doi.org/10.21595/jfocg.2021.22027

Check for updates

Copyright $(\mathbb{C} 2021$ Fabiana Augusto Novo Borghi, et al. This is an open access article distributed under the Creative Commons Attribution License, which permits unrestricted use, distribution, and reproduction in any medium, provided the original work is properly cited.

\begin{abstract}
Paralysis is the complete abolition of movement in a given territory of the body. Traumatic peripheral facial paralysis (PFPT) is characterized by lack of movement, due to injuries to the seventh cranial nerve (Facial Nerve), as a result of surgical resection of tumors in the region, firearm trauma, falls etc., can be uni or bilateral. Individuals affected by this paralysis when unilateral, tend to present facial asymmetry between the hemi faces, both at rest and in movement, as well as functional limitations to perform activities such as drinking, eating, speaking and problems in ocular hydration (dryness or watery eyes). In the case report, the patient underwent several types of treatment with very limited results. After 30 days of therapy with the hyperboloid, the patient reported improvements in clinical and motor conditions.
\end{abstract}

Keywords: facial nerve trauma, facial paralysis, facial nerve, exercises, proprioceptive stimuli.

\section{Introduction}

Facial paralysis (PF) can be central or peripheral.

Central PF is an injury to the seventh cranial nerve (Facial Nerve), which occurs in its intracranial path and can be caused by stroke, tumors, HIV, diabetes, etc. Loss of movement reaches the lower third of one side of the face and can affect the arms and legs on the opposite side. The patient performs the movements of the face, but the mouth deviates to the healthy side, which makes it difficult to articulate words and chew.

Unilateral peripheral PF is an injury that occurs below the facial nerve nucleus in the brain stem and compromises all facial mimic movements on that side. The patient with unilateral peripheral facial palsy has facial asymmetry, erasure of the nasolabial fold and wrinkles on the forehead, slow, reduced and incomplete blinking and deviation of the oral rhyme to the normal side. Upon ocular closure, lagophthalmos (inability to close the eye) and the Bell signal (upward deviation of the globe) are observed. There may be symptoms such as: facial pain, paraesthesias in the affected hemiface and neck, hypo or hyperlacrimation, changes in salivation, taste, hearing, vertigo and tinnitus.

About $50 \%$ of the population affected by PFP, the etiology is unknown, being diagnosed as idiopathic or Bell's being the first cause, with an incidence of 20 cases per 100,000 inhabitants and the second cause is traumatic PF.

The facial nerve (NF) is sensitive and motor, it is responsible for facial expression. Presenting a motor root and a sensory, taste and sensory root.

The trunks of the motor fibers pass through the parotid gland reaching the face, where they give two main branches: the facial and cervico facial branches, which branch out in a fan to innervate the cutaneous muscles of the face and neck.

Some motor fibers go to the hyoid style muscle and to the posterior belly of the digastric.

The sensory fibers (gustatory) follow a branch of the NF, which is the chord of the eardrum that will join the lingual nerve (mandibular branch, third branch of the trigeminal nerve), becoming a vector to be distributed in the two anterior thirds of the tongue. 
The NF also has vegetative fibers (parasympathetic) that use the intermediate nerve and then go through the greater petrous nerve or the tympanic cord (both branches of the NF) to innervate the lacrimal, nasal and salivary glands (sublingual and submandibular gland).

In the literature, the treatment of traumatic peripheral facial paralysis is through an anastomosis surgical maneuver, transposition of the anterior temporal muscle, grafts, physiotherapy and speech therapy and botulinum toxin on the healthy side.

The hyperboloid is a hyperbolic silicone, which was officially launched in 1989 at the 4th Congress of Aesthetics in Caxambu - Minas Gerais. This is silicone with 35 Shore hardness, equivalent to the consistency of hard, raw and fibrous foods, providing the teeth, periodontium and mucosa with the activation of genes providing the tissue response.

The technique used in the case described was idealized by Afrânio Pereira Cheida [1], a dental surgeon from the city of Santa Bárbara d'Oeste - SP- Brazil.

\section{Clinical case report}

Results of after 30 days, the following results were observed.

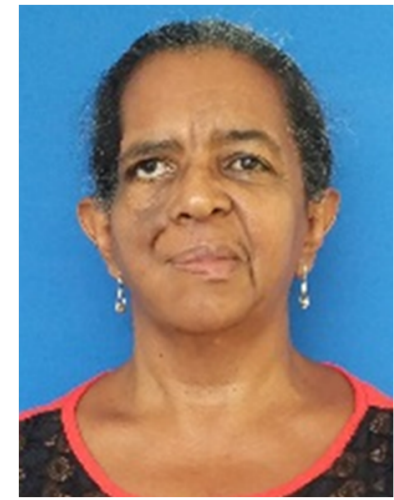

Fig. 1. Initial photo, March 2018

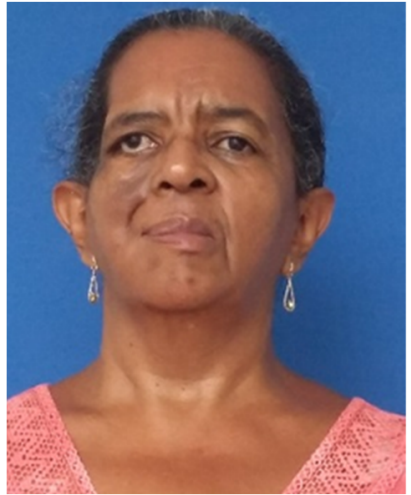

Fig. 2. Result of the exercise protocol for facial paralysis in 30 days of therapy, if trace a midlinewe check the difference before and after, April 2018

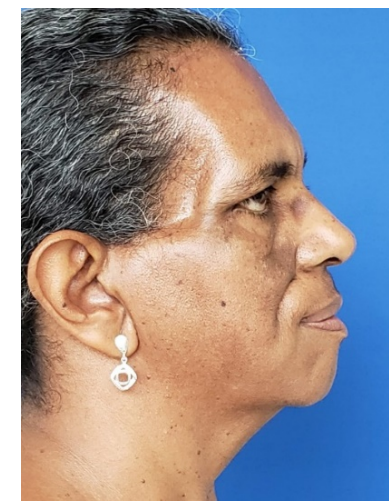

Fig. 3. Aspect of temporal muscle transposition surgery

According to information collected from the patient herself, and observed by the professional, there were changes in speech, swallowing, chewing, aesthetics and significant responses in the patient's quality of life.

The patient reported improvement in lacrimal dryness, bringing balance to this gland and also to the salivary gland, providing better quality chewing and swallowing. Having the possibility to 
throw the food on the paralyzed and healthy sides bringing comfort during meals and the patient's possibility of socialization, and an increase in self-esteem.

\section{Discussion}

According to Guyton (2006) [2] and Ferreira (2001) [5], the nerve injuries that cause PFPT are of multiple etiologies. The lesions may be complete and may cause a decrease and consequent loss of movement, muscle flaccidity and absence of facial wrinkles or be incomplete with signs and symptoms that vary according to the affected muscles.

They are classified according to the aggression suffered:

Neuropraxia: the structure of the nerve remains integral, there is no loss of axonal continuity between the neuron and the muscle, however there is an interruption of nerve conduction exclusively in the myelin sheath due to compression and consequently ischemia that makes it impossible the propagation of the action potential, however, the nerve, although paralyzed, conducts impulses above and below the lesion and responds to stimuli due to its reversibility. (Bruggermann et al, 2008) [3].

Axonotmesis: there is a loss of axon continuity due to axonal degeneration.

Neurotmesis: the lesion affects the axon, the endoneurium and the myelin sheath, and its regeneration is complex, due to the fact that neurons do not survive this type of injury, so the nerve will need to receive new adjacent nerve endings. (SALES, 2010) [4].

The remyelination process occurs with the proliferation of Schwann cells, which are responsible for the production of the new myelin layer along the axon of the demyelinated nerve, however the new nerve formation occurs with shorter intermodal segments than the original ones, making it slower driving speed. (FERREIRA, 2001) [5].

James T. Heaton, et al 2014 [6], in the study of the movement of rat whiskers after facial nerve injury, found evidence of autonomic contraction of the skeletal muscle. The study provided the first behavioral and anatomical evidence of spontaneous skeletal muscle recovery after motor nerve injury, which not only has implications for the interpretation of the results of facial nerve reinnervation, but also questions whether autonomic mediated innervation of the striated muscle occurs. naturally in other forms of neuropathies.

Pereira et al [7], in a systematic literature review, evaluated the influence of facial exercises on the recovery of individuals with peripheral facial paralysis and through a meta analysis concluded that facial exercises can improve the functionality of the affected subjects.

Guedes, Zelita Caldeira Ferreira in 1994 [8] reports that the mechanical stimulation of the facial musculature reduces the sequelae caused by paralysis.

Roberto S. Carvalho et all in Charles McNeill 2000 [9] concluded in his research: "the mechanically stimulated response, in vivo, seems to involve neural, vascular and physical phenomena, which should probably be combined to explain the extent of cell activation by mechanical stimulation".

The hyperboloid, an instrument of mechanical stimulation of muscles and salivary glands, with its hyperbolic shape provides greater performance with little energy expenditure, resulting in tissue response during its use. (Cheida, Afrânio Pereira, 2004) [1].

In the case presented, we observed a functional and aesthetic improvement, which generated a good degree of satisfaction.

\section{Exercise protocol}

1) Chewing $-3 x$ daily.

3 minutes on the paralyzed side.

1 minute and 30 seconds on the unaffected side.

3 minutes alternating unilateral.

2) Toning of the buccinator. 
3 minutes on the paralyzed side.

1 minute and 30 seconds on the unaffected side.

3 ) Toning the orbicularis lateralis.

3 minutes on the paralyzed side.

1 minute and 30 second side unaffected.

4) Stimulation of the tongue with elastic.

3 movements.

5) Stimulation of the tongue with hyperboloid.

3 movements.

6) Orthodontic pacifier.

3 minutes of suction.

7) Pacifier with hyperboloid.

3 minutes of suction.

8) Hold your eyes tight.

Count to 10.

9) Raise your eyebrow with your eyes closed.

Count to 10.

\section{Conclusions}

The protocol of prescribed exercises proved to be effective and with good results in PFPT, acting in the reestablishment of vegetative functions: swallowing, speech and chewing.

Clinical application: patients with traumatic peripheral facial paralysis and idiopathic facial paralysis.

\section{References}

[1] Cheida Afrânio Pereira, Hyperboloid masticating instrument. 1st edition, (in Portuguese), 2004.

[2] A. C. Guyton and J. E. Hall, Treaty of Medical Physiology. 11th edition, (in Portuguese), Rio de Janeiro, Brazil: Saraiva, 2006

[3] K. Bruggemann, Physiotherapy in Neurology. (in Portuguese), São Paulo, Brazil: Santos, 2009.

[4] Sales et al., Peripheral Facial Paralysis: Etiology, Symptoms and Physiotherapeutic Treatment. 2010.

[5] S. Edshage, "Peripheral nerve injuries--diagnosis and treatment," (in Portuguese), New England Journal of Medicine, Vol. 278, No. 26, pp. 1431-1436, Jun. 1968, https://doi.org/10.1056/nejm196806272782606

[6] J. T. Heaton et al., "Rat whisker movement after facial nerve lesion: Evidence for autonomic contraction of skeletal muscle," Neuroscience, Vol. 265, pp. 9-20, Apr. 2014, https://doi.org/10.1016/j.neuroscience.2014.01.038

[7] L. Pereira, K. Obara, J. Dias, M. Menacho, E. Lavado, and J. Cardoso, "Facial exercise therapy for facial palsy: systematic review and meta-analysis," (in Portuguese), Clinical Rehabilitation, Vol. 25, No. 7, pp. 649-658, Jul. 2011, https://doi.org/10.1177/0269215510395634

[8] Guedes and Zelita Caldeira Ferreira, The situation of speech therapy in the multidisciplinary team of patients with peripheral facial paralysis. (in Portugueses), São Paulo, 1994.

[9] Sharles Mcneill, Science and practice of occlusion. 1st edition, (in Portuguese), São Paulo, Brazil: Quintensence, 2000.

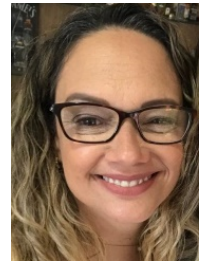

Fabiana Augusto Novo Borghi, Dentist, Specialist in Functional Jaw Orthopedics, Member of the Brazilian Academy of Functional Jaw Orthopedics, member of the study group IVO - Institutu Vitae Onmiscentia Brasil, Italy, volunteered at the Facial Outpatient Clinic at UNIFESP - EPM years 2018 and 2019, Santa Bárbara d' Oeste, SP, Brazil. 
TREATMENT OF TRAUMATIC FACIAL PARALYSIS WITH MECHANICAL STIMULUS - HYPERBOLOID: CASE REPORT.

FABIANA Augusto Novo Borghi, Rosana QueIROZ, José RICARDo GuRGEL TESTA

Rosana Queiroz, dental surgeon, specialist in Functional Maxillary Orthopedics, master's in health sciences, Ph.D. student in the Postgraduate Program in Otorhinolaryngology and Head and Neck Surgery at UNIFESP - EPM, São Paulo, SP, Brazil.

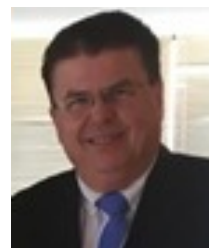

José Ricardo Gurgel Testa, Physician, Adjunct Professor of Pediatric Otorhinolaryngology at Unifesp - EPM, São Paulo, SP, Brazil. 